\title{
Open Turbulent Image Set (OTIS)
}

\author{
Jérôme Gilles ${ }^{\mathrm{a}, * *}$, Nicholas B. Ferrante ${ }^{\mathrm{a}}$ \\ ${ }^{a}$ San Diego State University - Department of Mathematics $\mathcal{E}$ Statistics, 5500 Campanile Dr, San Diego, CA 92182, USA
}

\begin{abstract}
Long distance imaging is subject to the impact of the turbulent atmosphere. This results into geometric distortions and some blur effect in the observed frames. Despite the existence of several turbulence mitigation algorithms in the literature, no common dataset exists to objectively evaluate their efficiency. In this paper, we describe a new dataset called OTIS (Open Turbulent Images Set) which contains several sequences (either static or dynamic) acquired through the turbulent atmosphere. For almost all sequences, we provide the corresponding groundtruth in order to make the comparison between algorithms easier. We also discuss possible metrics to perform such comparisons.
\end{abstract}

(c) 2016 Elsevier Ltd. All rights reserved.

\section{Introduction}

Turbulence mitigation algorithms aiming to restore a clean image from a set of distorted observations has been widely studied since more than a decade. See for instance Frakes et al. (2001); Gilles et al. (2008); Mao and Gilles (2012); Lou et al. (2013); Anantrasirichai et al. (2013); Halder et al. (2013); Micheli et al. (2013); Gal et al. (2014) just to cite a few. Most contributions are based on the common idea of combining stabilization and deconvolution steps and they only differ on the techniques used in their implementation. Most of the time, each author has access to data which are not freely available hence making the comparison difficult. It becomes necessary to build an open dataset of images as well as select some metric which can be used by the community in order to get an objective comparison of the different developed algorithms.

The purpose of this paper is to propose such open dataset. It is made of a collection of sequences of static scenes as well as dynamic scenes (i.e with a moving target). Most of the static sequences correspond to the observation of printed charts. Such approach permits to create a groundtruth associated to each sequence and then can be used by some metric to assess the reconstruction efficiency.

We want to emphasize that the purpose of this dataset is not to assess the turbulence itself but the visual enhancements provided by mitigation algorithms. Therefore, we do not provide any physical measurements (temperature, wind, $C_{n}^{2}, \ldots$ )

\footnotetext{
${ }^{* *}$ Corresponding author: Tel.: +1-619-594-7240; fax: +1-619-594-6746; e-mail: jgilles@mail.sdsu. edu (Jérôme Gilles)
}

and we categorized the observed turbulence as either "weak", "medium" or "strong".

The paper is organized as follows. Section 2 presents the equipment used and the acquisition procedure. The acquired sequences are described in section 3. Possible evaluation metrics are discussed in section 4 and section 5 concludes this paper.

\section{Equipment and acquisition procedure}

\subsection{Equipment}

All sequences were acquired with a GoPro Hero 4 Black camera modified with a RibCage Air chassis permitting to adapt several type of lenses. We always used a $25 \mathrm{~mm}, \mathrm{f} / 2.014 \mathrm{~d}$ HFOV 3MP lens. The camera was setup at a 1080p resolution and a framerate of 24 frames per second (fps). A small tripod was used to hold the camera (see Figure 1). The camera was controlled by the standard GoPro App on a Samsung Galaxy tablet.

The acquired sequences contain both natural elements from the observed scene as well as artificial "targets". For the static sequences, we used two charts containing some geometric patterns at different spatial frequencies and orientations (see Figure 2). These charts were printed on a poster (each chart has a size of $35 \times 35 \mathrm{~cm}$ ) and held up by a homemade wooden stand. For the dynamic sequences, we used a standard remote controlled car (see Figure 3).

\subsection{Procedures}

All acquisitions were made on hot sunny days in order to guaranty a certain amount of atmospheric turbulence. All 


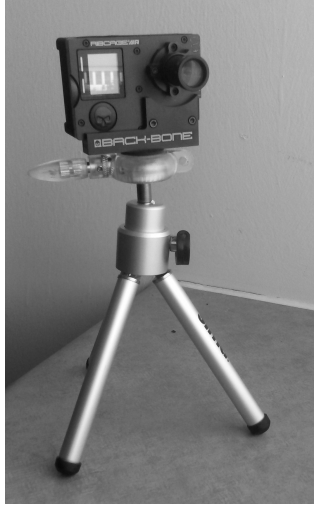

Fig. 1: RibCage Air Modified GoPro Hero 4 Black camera with a 25mm f/2.0 14d HFOV 3MP lens on its tripod.

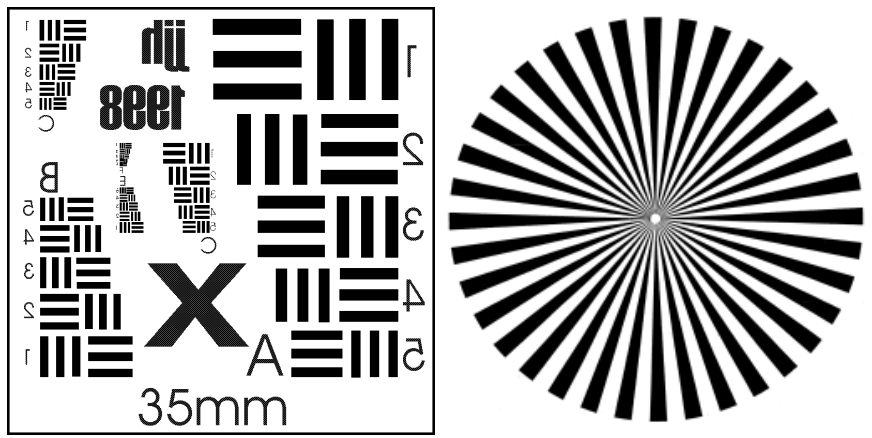

Fig. 2: The two charts serving as our static artificial targets after being printed on a poster.

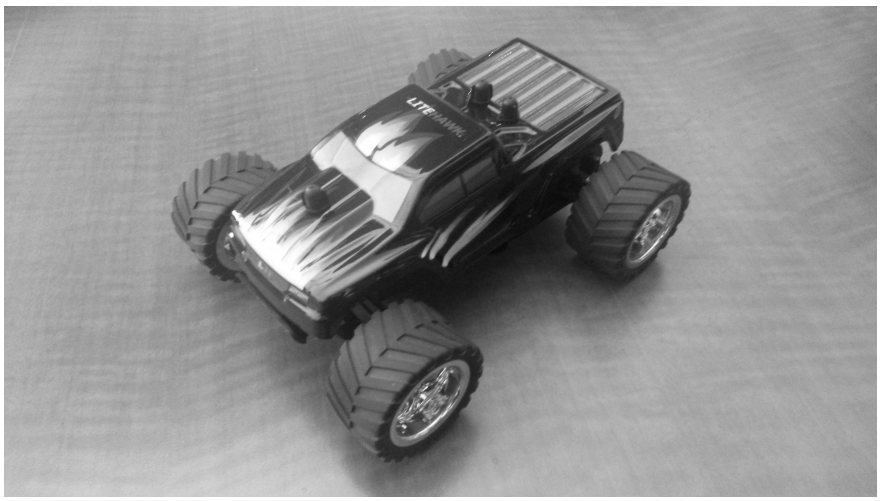

Fig. 3: Remote Controlled car utilized as our moving target for the dynamic sequences.

equipments were setup on a practice field at the San Diego State University. This field is equipped with artificial turf which reflects very well the sun heat, leading to high level of turbulence. The camera stood at about $10 \mathrm{~cm}$ above the ground observing the target positioned at several distances.

After all acquisitions were done, the different recorded movies were downloaded on a Linux computer and split into sequences of PNG image files using the ffmpeg command ${ }^{1}$. The different region of interest are finally cropped via the convert command

\footnotetext{
${ }^{1}$ https://ffmpeg.org/
}

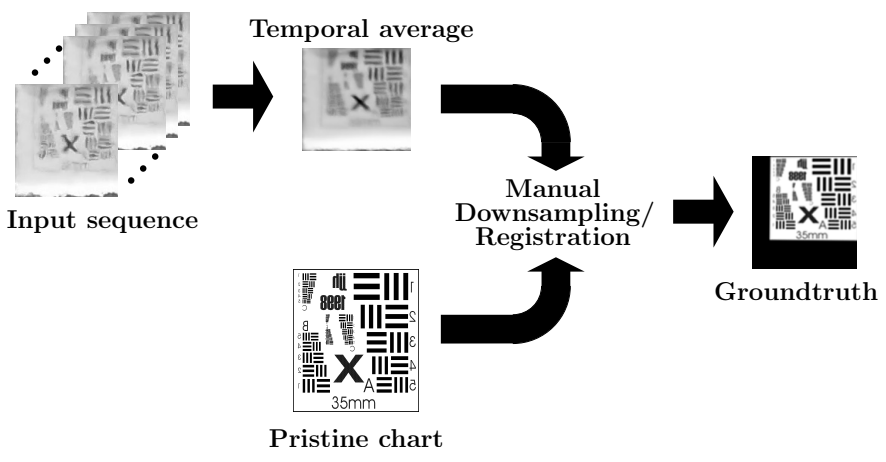

Fig. 4: Groundtruth images creation procedure.

(from the imagemagick library ${ }^{2}$ ) and saved as individual PNG sequences. Since the Matlab $®$ software is widely used by the community, we also provide each sequence saved as a Matlab 3D matrix (the first two coordinates are the spatial coordinates while the third one corresponds to time) save in a .mat file.

Since the purpose of this dataset is to be used for evaluating turbulence mitigation algorithms, all sequences containing the two above mentioned charts are provided with a groundtruth image. This groundtruth image contains the pristine chart after being downsampled and registered to the actual sequence. In practice, we manually registered the pristine chart on a temporal average of the input sequence using the GIMP ${ }^{3}$ software (this procedure is summarized in Figure 4).

The dynamic sequences are also provided with their respective groundtruths. Each groundtruth is a sequence of binary images containing the bounding box corresponding to the moving target position. These groundtruths were created with the software Sensarea ${ }^{4}$.

\section{Collected data}

The different available sequences were acquired between June 18th and August 16th, 2016.

\subsection{Static sequences}

OTIS contains a total of seventeen static sequences. One provides the observation of natural elements in the scene (doors, steps, fence) and the sixteen remaining ones are made of the two previous charts. The former ones are provided with their corresponding groundtruth images in order to facilitate future algorithm evaluations. The complete list of all static sequences as well as their characteristics is given in Table 1. Several samples from different fixed pattern sequences as well as with their corresponding groundtruths are illustrated in Figure 5.

\subsection{Dynamic sequences}

OTIS contains four dynamic sequences. Each sequence contains a moving remote controlled car at different distances and

\footnotetext{
${ }^{2}$ http://www. imagemagick.org/

${ }^{3}$ https://www.gimp.org/

${ }^{4}$ http://www.gipsa-lab.grenoble-inp.fr/ pascal.bertolino/ bin/download.php?file=sensarea.exe
} 


\begin{tabular}{|l|l|l|l|l|l|}
\hline Folder Name & $\begin{array}{l}\text { Sequence } \\
\text { Name }\end{array}$ & $\begin{array}{l}\text { Number } \\
\text { of images }\end{array}$ & Image size & $\begin{array}{l}\text { Turbulence } \\
\text { level }\end{array}$ & $\begin{array}{l}\text { Ground } \\
\text { Truth }\end{array}$ \\
\hline Fixed Background & Door & 300 & $520 \times 520$ & Strong & No \\
\hline Fixed Patterns & Pattern1 & 64 & $302 \times 309$ & Weak & Yes \\
& Pattern2 & 64 & $291 \times 287$ & Weak & Yes \\
& Pattern3 & 300 & $113 \times 117$ & Strong & Yes \\
& Pattern4 & 300 & $109 \times 113$ & Strong & Yes \\
& Pattern5 & 300 & $113 \times 117$ & Strong & Yes \\
& Pattern6 & 300 & $109 \times 113$ & Strong & Yes \\
& Pattern7 & 300 & $122 \times 125$ & Strong & Yes \\
& Pattern8 & 300 & $119 \times 122$ & Strong & Yes \\
& Pattern9 & 300 & $152 \times 157$ & Medium & Yes \\
& Pattern10 & 300 & $149 \times 149$ & Medium & Yes \\
& Pattern11 & 300 & $172 \times 183$ & Medium & Yes \\
& Pattern12 & 300 & $172 \times 173$ & Medium & Yes \\
& Pattern13 & 300 & $202 \times 202$ & Weak & Yes \\
& Pattern14 & 300 & $196 \times 193$ & Weak & Yes \\
& Pattern15 & 300 & $134 \times 139$ & Strong & Yes \\
& Pattern16 & 300 & $135 \times 135$ & Strong & \\
\hline
\end{tabular}

following different trajectories. Our goal was to create both sequences where the moving car remains easy to detect (even if it is affected by geometric distortions), as well as sequences where the car movement magnitude is close to the magnitude of the turbulence hence making them more challenging to detect. The complete list of all available sequences as well as their characteristics are given in Table 2. Two frames from a dynamic sequence with their corresponding bounding boxes are given in Figure 6.

\section{Quality metric}

The main intent of OTIS is to facilitate the comparison of turbulence mitigation algorithms. Having test sequences as well as their groundtruth images is not sufficient to design a complete evaluation process. Indeed, it remains to choose some metrics in order to obtain an objective comparison. Denoting $I_{R}$ and $I_{G T}$ the restored and groundtruth images, respectively, the most used metric to compare images is the Mean Square Error (MSE) defined by

$$
\operatorname{MSE}\left(I_{R}, I_{G T}\right)=\frac{1}{N} \sum_{x}\left(I_{R}(x)-I_{G T}(x)\right)^{2},
$$

where $N$ is the number of pixel in the image. Despite its popularity, the MSE has several drawbacks, notably it doesn't take into account the geometric information contained within the image. The Structural Similarity Index Measure (SSIM) was proposed by Wang et al. (2004) to circumvent these issues. The SSIM is a perception-based model which consider structural (geometrical) distortions in the image. Given the fact that one of the major degradation due to the turbulence is the geometrical distortions, the SSIM metric appears to be the most adapted metric to compare turbulence mitigation algorithms.
Regarding the assessment of tracking algorithms applied on the dynamic sequences, the methodology developed in Gilles et al. (2010) can be used.

\section{Conclusion}

In this paper, we described a new publicly available dataset called OTIS (Open Turbulent Image Set) of sequences acquired through atmospheric turbulence which purpose is to aid at the assessment of mitigation algorithms. This dataset contains both static and dynamic sequences with groundtruth images. We propose OTIS in several versions: in color or grayscales and either saved as a set of PNG images or as 3D Matlab matrices. These different versions of OTIS can be downloaded for free at https://zenodo.org/communities/otis/. We also suggest the use of the SSIM metric to perform such objective performance evaluations.

\section{Acknowledgments}

This work was supported by the NSF grant DMS-1556480, the San Diego State University Presidential Leadership Funds and the Air Force Office of Scientific Research grant FA955015-1-0065.

\section{References}

Anantrasirichai, N., Achim, A., Kingsbury, N.G., Bull, D.R., 2013. Atmospheric turbulence mitigation using complex wavelet-based fusion. IEEE Transaction in Image Processing 22, 2398-2408.

Frakes, D.H., Monaco, J.W., Smith, M.J.T., 2001. Suppression of atmospheric turbulence in video using an adaptive control grid interpolation approach. IEEE Conference Proceedings on Acoustics, Speech and Signal Processing $3,1881-1884$ 
Table 2: Summary of the different dynamic sequences

\begin{tabular}{|l|l|l|l|l|l|}
\hline Folder Name & $\begin{array}{l}\text { Sequence } \\
\text { Name }\end{array}$ & $\begin{array}{l}\text { Number } \\
\text { of images }\end{array}$ & Image size & $\begin{array}{l}\text { Turbulence } \\
\text { level }\end{array}$ & $\begin{array}{l}\text { Ground } \\
\text { Truth }\end{array}$ \\
\hline Moving Target & Car1 & 100 & $200 \times 200$ & Medium & Yes \\
& Car2 & 315 & $500 \times 200$ & Medium & Yes \\
& Car3 & 51 & $300 \times 300$ & Medium & Yes \\
& Car4 & 101 & $300 \times 300$ & Medium & Yes \\
\hline
\end{tabular}
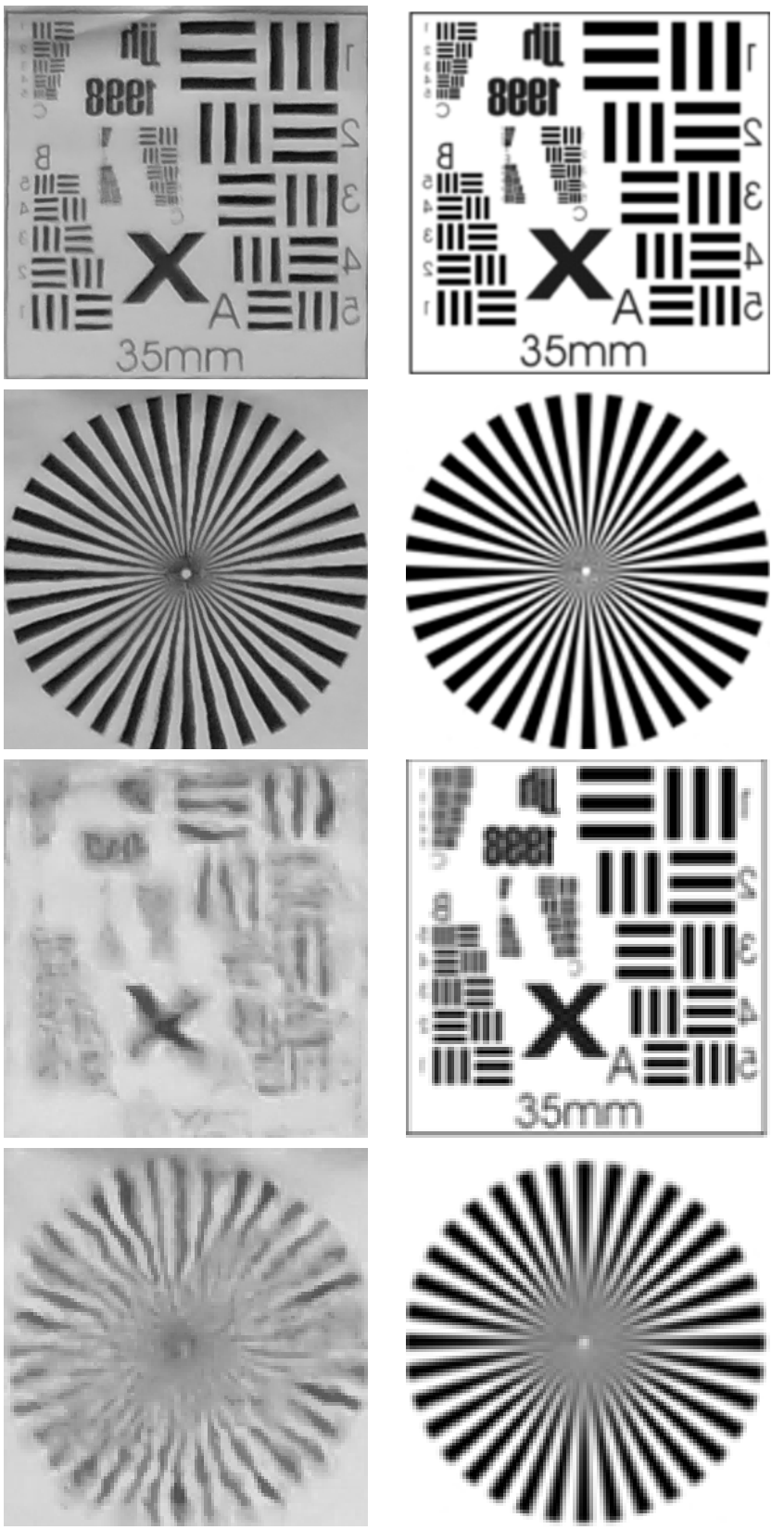

Fig. 5: Samples of frames from different fixed pattern sequences (left) and their corresponding groundtruths (right). The first two rows correspond to a weak turbulence case while the last two ones correspond to a strong turbulence case.

Gal, R., Kiryati, N., Sochen, N., 2014. Progress in the restoration of image sequences degraded by atmospheric turbulence. Pattern Recognition Letters
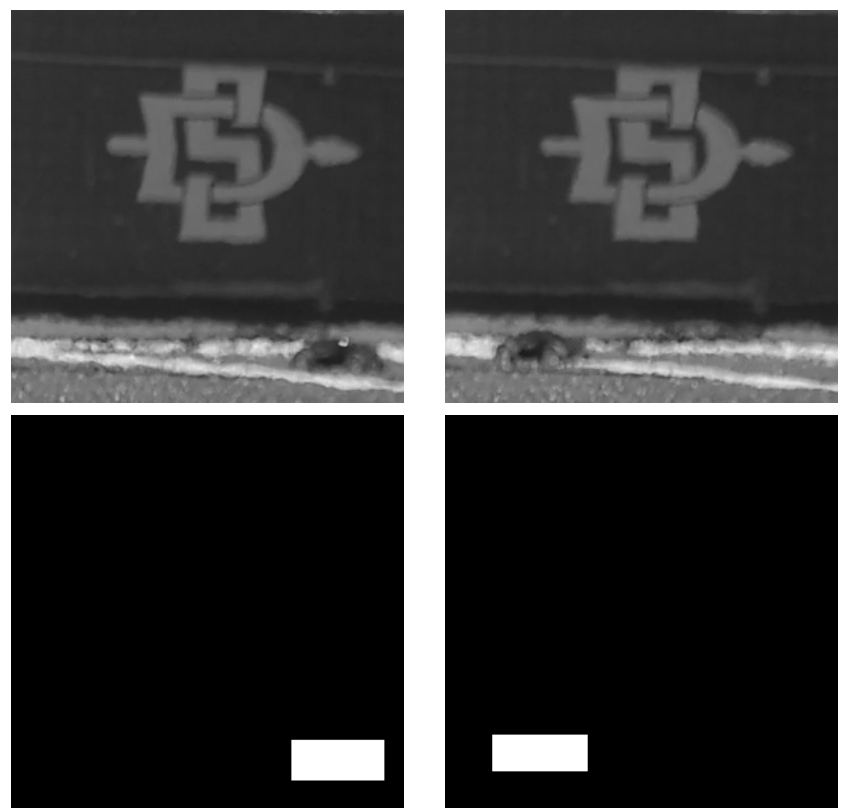

Fig. 6: Frames 16 and 34 from a dynamic sequences (top) and their corresponding groundtruths frames (bottom).

$48,8-14$.

Gilles, J., Dagobert, T., Franchis, C.D., 2008. Atmospheric turbulence restoration by diffeomorphic image registration and blind deconvolution, in: Advanced Concepts for Intelligent Vision Systems - 10th International Conference, ACIVS 2008, Proceedings, Springer, Juan-les-Pins, France. pp. 400409.

Gilles, J., Landeau, S., Dagobert, T., Chevalier, P., Stiée, E., Diaz, D., Maillart, J.L., 2010. METRIC: a complete methodology for performances evaluation of automatic target detection, recognition and tracking algorithms in infrared imagery, in: OPTRO 2010, Paris, France.

Halder, K., Tahtali, M., Anavatti, S., 2013. A fast restoration method for atmospheric turbulence degraded images using non-rigid image registration, in: 2013 International Conference on Advances in Computing, Communications and Informatics (ICACCI), Mysore. pp. 394-399.

Lou, Y., Kang, S.H., Soatto, S., Bertozzi, A.L., 2013. Video stabilization of atmospheric turbulence distortion. Inverse Problems and Imaging 7, 839861.

Mao, Y., Gilles, J., 2012. Non rigid geometric distortions correction - application to atmospheric turbulence stabilization. Journal of Inverse Problems and Imaging 6, 531-546.

Micheli, M., Lou, Y., Soatto, S., Bertozzi, A.L., 2013. A linear systems approach to imaging through turbulence. Journal of Mathematical Imaging and Vision 48, 185-201.

Wang, Z., Bovik, A., Sheikh, H., Simoncelli, E., 2004. Image quality assessment: from error visibility to structural similarity. IEEE Trans on Image Processing 13, 600-612. 